\title{
Mortalite A La Phase Aiguë De L'avc Hemorragique Sous Notre Protocole De Baisse De La Tension Arterielle A l'Hopital Universitaire (HU) Befelatanana Antananarivo
}

\author{
Rajaonarison Lala Andriamasinavalona, MD \\ Rasaholiarison Nomena Finiavana, MD \\ Razafindrasata Ratsitohara Santatra, MD \\ Razafimahefa Julien, MD \\ Zodaly Noël, MD \\ Tehindrazanarivelo Alain Djacoba, PhD
}

Faculté de Médecine d'Antananarivo,

Université d'Antananarivo, Madagascar

Doi: 10.19044/esj.2018.v14n18p325 URL:http://dx.doi.org/10.19044/esj.2018.v14n18p325

\section{Abstract}

Introduction: Hemorrhagic stroke can be seen in $90 \%$ of cases with high blood pressure. It is a predictor of intra-hospital mortality. Our goal is to compare the mortality rate based on admission under our therapeutic strategy for HTA in the acute phase of stroke. Method: A retrospective descriptive, cross-sectional study of consecutive patients was conducted over a 12-month period during the year 2017. Results: We had 20\% (3/15) mortality rate in acute phase in those with systolic blood pressure (SBP) in admission at $\geq 180$ $\mathrm{mmHg}$ (group II) and no death (0/17) was recorded in the those with SBP in admission at $140-179 \mathrm{mmHg}$ (group I). A SBP at the 7th day (D7) less than $140 \mathrm{mmHg}$ was not rich in those two groups: $35.29 \%$ in group $\mathrm{I}(\mathrm{n}=6)$ and $46.66 \%$ in group II $(n=7)$ respectively. The sex ratio was 1 . High blood pressure remains the main cardio-vascular risk factor reported in our study (81.21\%). All our patients had Glasgow scale value between 13-15 and only the NIHSS value $>10$ was associated with high blood pressure in acute phase $(\mathrm{p}=0.0131)$. Brain CT Scan was realised in majority of cases at the second day (D2) of stroke $(n=16)$. This, however, is with localisation preference in the basal ganglia, with intra-cerebral hemmorhage (ICH) scale at 1 in $65.62 \%$ $(n=21)$ of cases. Conclusion: Intra-hospital mortality in acute phase of hemorrhagic stroke remains high. Instauration of stroke field in Madagascar is necessary and this gives the Befelatanana University Hospital a Neuro- 
Vascular Intensive Care Unit too.

Keywords: Blood Pressure, ICH scale, Madagascar, Mortality, Stroke

\section{Résumé}

L'Accident Vasculaire Cérébral (AVC) hémorragique est associé dans $90 \%$ des cas à une élévation de la tension artérielle, qui est un des facteurs prédictifs de mortalité intra hospitalière. Notre objectif est de comparer la mortalité selon la TA (Tension Artérielle) au moment de l'admission sous notre stratégie thérapeutique devant l' HTA (Hyper Tension Artérielle) à la phase aiguë de l'AVC hémorragique. Une étude rétrospective descriptive, transversale sur dossier des patients consécutifs fut menée sur une période de 12 mois durant l'année 2017. Nos résultats ont rapporté $20 \%$ de mortalité à la phase aiguë pour ceux avec TAS à l'admission $\geq 180 \mathrm{mmHg}$ versus aucun décès dans le groupe des TAS à l'admission entre 140-179mmHg. Une TAS à $\mathrm{J} 7$ de moins de $140 \mathrm{mmHg}$ n'a pas été atteinte dans les deux groupes. Cela montre que l'HTA reste le facteur de risque le plus rapporté (81.21\%). Tous nos patients ont eu un score de Glasgow entre 13-15 et seule un score NIHSS > 10 est associé à l'HTA à la phase aiguë $(\mathrm{p}=0.0131)$. Le scanner cérébral a été réalisé dans la majorité des cas à $\mathrm{J} 2(\mathrm{n}=16)$, avec une localisation préférentielle au niveau des noyaux gris centraux et un score ICH à 1 dans $65.62 \%(n=21)$. Ces résultats avèrent que la mortalité intra hospitalière à la phase aiguë de l'AVC hémorragique reste élevée. De même, il est nécessaire de mettre en place la filière AVC à Madagascar et de munir 1' HU Befelatanana d'une Unité de Soins Intensifs en Neurologie Vasculaire.

Mots-clés : Accident Vasculaire Cérébral, Madagascar, Mortalité, Score ICH, Tension Artérielle

\section{Introduction}

L'accident vasculaire cérébral (AVC) se définit comme un déficit neurologique d'installation brutale et d'allure vasculaire présumée (Vuillier et al., 2004). Il est de nature hémorragique quand il fait suite à une extravasation de sang dans le parenchyme cérébral. Les AVC hémorragiques représentent 10 à $15 \%$ des AVC, soient 10 à 30 cas par 100000 habitants (Feigin et al., 2003 ; Qureshi et al., 2001). Leur mortalité à 30 jours est de 30 à 55\% des cas dont la moitié se rencontre à la phase aiguë, spécialement dans les premières 48h (Balami et al., 2012). A Madagascar, une étude en population générale menée à Antananarivo a montré que la prévalence annuelle de l'AVC est 1,6 fois plus élevée chez les Malagasy par rapport aux Français, 1,25 fois plus élevée par rapport aux Américains blancs et 1,30 fois plus élevée par rapport aux Martiniquais. L'AVC constitue aussi la première cause de mortalité 
hospitalière (21\%) (OMS, 2013). Une étude de Ravelojaona FH et al en 2012 dans le service de neuropsychiatrie de l'HU Befelatanana a rapporté 78.8\% d'AVC ischémique et $22.20 \%$ d'AVC hémorragique. Le volume de l'hémorragie cérébrale est un des facteurs prédictifs de mortalité intra hospitalière et un des seuls modifiables (Stapf et al., 2015). Ceci est rattaché à l'hypertension artérielle (HTA) qui est reconnue comme le facteur aggravant de l'hémorragie, de l'importance du volume de l'hématome et des épisodes de resaignement (Steiner et al., 2006). Selon les données de la littérature 70\% des patients présentent une expansion hémorragique dans les 24 premières heures (Davis et al., 2006). Aussi que dans $90 \%$ des cas, les patients ont une élévation tensionnelle à la phase aiguë de l'AVC (Qureshi, 2013).

En revanche, la baisse agressive de la tension artérielle (TA) à la phase aiguë n'aggrave pas l'état clinique des patients (Stapf et al., 2015). Des études ont montré une corrélation significative entre réduction de la pression artérielle à la phase aiguë de l'AVC hémorragique et la réduction de la croissance de l'hématome (Ohwaki et al., 2004). La Société Française Neurovasculaire recommande une baisse de la Tension Artérielle systolique (TAS) inférieure à $140 \mathrm{mmHg}$ en soixante minutes pour un AVC hémorragique, et en moins de $6 \mathrm{~h}$ pour une TAS comprise entre $150-220 \mathrm{mmHg}$. Cependant, à Madagascar documenter la nature de l'AVC à la phase aiguë reste un grand problème pour plusieurs raisons. Dans ce cas, appliquer les recommandations internationales sur la gestion de la TA à la phase aigüe de l'AVC hémorragique est limité. C'est ainsi qu'on voudrait décrire notre stratégie thérapeutique devant l' HTA à la phase aiguë de l'AVC hémorragique des patients ayant été hospitalisés auprès du service de Neurologie du CHU JRB, puis d'en rapporter le taux de mortalité au troisième et septième jour de leur AVC par rapport aux chiffres tensionnels systoliques à l'admission et les caractéristiques cliniques des patients.

\section{Matériels et méthode}

Il s'agit d'une étude descriptive transversale et analytique, sur une période de 12 mois du 01 Janvier 2017 jusqu'au 31 Décembre 2017, qui vise à décrire notre stratégie thérapeutique pour baisser l'hypertension artérielle (HTA) à la phase aiguë d'un AVC hémorragique et de rapporter la survie des patients à $\mathrm{J} 3$ et $\mathrm{J} 7$ auprès de l'USFR de Neurologie HU Befelatanana Antananarivo Madagascar. Nous avons inclus tout individu ayant été hospitalisé et diagnostiqué comme AVC hémorragique avec réalisation d'un scanner cérébral confirmant la nature anatomique lésionnelle auprès de l'USFR de Neurologie, aussi bien que tout individu disposant de données cliniques de $\mathrm{J} 1$ à $\mathrm{J} 7$ de leur $\mathrm{AVC}$, mentionnant la valeur de la TA à $\mathrm{J} 1$ de leur $\mathrm{AVC}$ avec leur score NIHSS qui a été calculé à partir de l'observation clinique faite auprès des Services des urgences HU Befelatanana. Tout dossier 
incomplet ou patient présentant un ramollissement hémorragique de son AVC ischémique a été exclu. Nous avons défini comme AVC hémorragique un déficit neurologique d'installation brutale, focalisé, et d'allure vasculaire présumée avec preuve tomodensitométrique (TDM cérébrale) attestant la présence de sang au sein du parenchyme cérébral. L' HTA à la phase aiguë est définie par une valeur de la TAS $\geq 140 \mathrm{mmHg}$. Les TA ont été mesurées à l'admission auprès du Service d'Accueil Triage et Urgence (ATU) HU Befelatanana et le matin à $\mathrm{J} 3$ et $\mathrm{J} 7$; la phase aiguë étant les sept premiers jours suivant l'AVC. Notre stratégie thérapeutique devant une HTA à la phase aiguë dans le service est la suivante: si le patient n'a pas encore de preuve tomodensitométrique du caractère hémorragique de l'AVC, nous utilisons le protocole Nicardipine IV si la TAS est $\geq 180 \mathrm{mmHg}$. La TAS cible est à < $180 \mathrm{mmHg}$ et peut être maintenue pendant les sept premiers jours tant que la TAS ne dépasse pas $180 \mathrm{mmHg}$. Le protocole est associé à une surveillance de la TA toutes les 15 minutes pendant la première heure, puis toutes les 30 minutes pendant la deuxième heure, toutes les heures pendant les 4 premiers jours de l'AVC, et de $\mathrm{J} 4$ à $\mathrm{J} 7$ une prise de la TA est effectuée le matin, le midi et le soir avant de se coucher sans prise d'anti-HTA orale. Dès que nous avons une preuve tomodensitométrique du caractère hémorragique de l'AVC, pendant les quatre premiers jours, la TAS cible est $<140 \mathrm{mmHg}$ avec le protocole Nicardipine. A partir de J4, nous introduisons une anti-HTA orale au choix du Médecin Neurologue responsable du patient en association ou non avec la Nicardipine IV si la TAS reste $\geq 180 \mathrm{mmHg}$. La TAS cible reste à moins de $140 \mathrm{mmHg}$.

Les paramètres étudiés dans notre étude sont la fréquence absolue et relative par rapport au genre, à l'âge du patient et au type d'AVC, et les caractéristiques cliniques de nos patients, lesquels ont été divisés en deux groupes selon la valeur de leur TAS à l'admission. Le premier groupe (groupe I) regroupe tous les patients avec une TAS entre $140-179 \mathrm{mmHg}$ et le second groupe (groupe II) regroupent tous les patients avec une TAS $\geq$ $180 \mathrm{mmHg}$.Autres paramètres étudiés sont la démographie, c'est-à-dire, l'âge et le genre; la symptomatologie englobant les facteurs de risque cardiovasculaire ; NIHSS et le score de Glasgow à l'entrée ; les caractéristiques scanographiques, c'est-à-dire, la localisation de la lésion, l'existence ou non de signe d'engagement tomodensitométrique, le Score $\mathrm{ICH}$ après la réalisation du scanner cérébral ; l'évolution de la courbe de la tension artérielle systolique prise le matin à $\mathrm{J} 3$ et $\mathrm{J} 7$ sous anti hypertenseur (intra veineuse jusqu'à $\mathrm{J} 4$ puis sous relais oral) ; la mortalité à $\mathrm{J} 3$ et à $\mathrm{J} 7$ durant la prise en charge ainsi que la force de liaison entre la baisse de la tension artérielle et le taux de mortalité à $\mathrm{J} 3$ et $\mathrm{J} 7$ en comparant le profil clinique et thérapeutique des patients avec un AVC hémorragique à la phase aiguë sur ceux qui sont décédés. Les données étaient collectées sur les dossiers médicaux des patients à l'aide d'un 
questionnaire préétabli. Pour le traitement des données et les tests statistiques, nous avons utilisé le logiciel $\mathrm{R}$ et l'association des variables selon le test de Fisher avec un seuil de significativité pour une valeur de $\mathrm{p}<0.05$. Les résultats sont représentés en valeur absolue, en pourcentage et en moyenne.

\section{Résultats}

Trente-deux patients sur 132 présentant AVC hémorragiques soient $24.24 \%$ et sur 729 patients hospitalisés soient $4.38 \%$ ont été retenus. Notre population d'étude représente $8.71 \%$ des cas par rapport au nombre total de patients hospitalisés et diagnostiqués comme un AVC avec ou sans preuve tomodensitométrique. Tous les patients ont eu un score de Glasgow $\geq 13$, et ont été transférés auprès du Service de Neurologie le jour même de leur passage auprès de 1'ATU HU Befelatanana. Seuls trois patients du groupe II ont eu un score NIHSS entre 16-20 (tableau I), douze patients ont eu une TAS entre $180-220 \mathrm{mmHg}$ et 3 patients ont eu une TAS $\geq 220 \mathrm{mmHg}$. L'objectif de la TAS à $\mathrm{J} 7$ de moins de $140 \mathrm{mmHg}$ n'a pas été atteint dans les deux groupes de population dans $35.29 \%$ des cas du groupe $I(n=6)$ et dans $46.66 \%$ des cas du groupe II $(n=7)$ (tableau II). Tous les patients du groupe II $(n=15)$, soit $46.87 \%$, ont été mis sous notre protocole Nicardipine IV et celui-ci était maintenu jusqu'à J4 pour $73.33 \%$ des patients $(n=11)$ et jusqu'à J7 pour $26.66 \%(\mathrm{n}=4)$ tant que la TAS était $\geq 180 \mathrm{mmHg}$. A partir de J4, l'administration de molécule anti HTA per os laissée au choix du Neurologue retrouvait dans $78.12 \%(n=25)$ une association de deux anti HTA ; les IEC (Inhibiteurs de l'Enzyme de Conversion) et inhibiteurs calciques ont été les plus utilisés. Trois patients du groupe II (20\%) ont succombé pendant la phase aiguë de leur AVC dont un patient avait eu une TAS à l'admission entre 180$220 \mathrm{mmHg}$ et les deux autres une TAS $\geq 220 \mathrm{mmHg}$ (Tableau I et II). 
Tableau I . Comparaison des 2 groupes selon les données démographiques

\begin{tabular}{|l|l|l|l|}
\hline & $\begin{array}{l}\text { Groupe I } \\
\text { n (\%) }\end{array}$ & $\begin{array}{l}\text { Groupe II } \\
\mathbf{n}(\%)\end{array}$ & Valeur p \\
\hline Genre & $07(41.17)$ & $09(60)$ & 0.749 \\
Masculin & $10(58.82)$ & $06(40)$ & \\
Féminin & $0(0)$ & $01(6.67)$ & \\
\hline Age (ans) & $04(23.53)$ & $06(40)$ & \\
$18-34$ & $10(58.82)$ & $05(33.33)$ & 0.864 \\
$35-49$ & $03(17.65)$ & $03(20)$ & \\
$50-64$ & & & 0.272 \\
$\geq 65$ & $13(76.47)$ & $13(86.67)$ & \\
& $01(5.89)$ & $01(6.67)$ & 0.0752 \\
Facteur de risque & $05(29.42)$ & $07(46.67)$ & \\
HTA & $06(35.30)$ & $06(40)$ & \\
Diabète & $03(17.65)$ & $03(20)$ & \\
Tabagisme & $03(17.65)$ & $07(46.67)$ & \\
Alcool & $04(23.53)$ & $04(26.67)$ & \\
AVC antérieur & & & \\
Risque) Facteurs de & 04 & & \\
$\geq 3$ FDR & & & \\
\hline
\end{tabular}

Tableau II. Comparaison des 02 groupes selon les données cliniques et scannographiques

\begin{tabular}{|l|l|l|l|}
\hline & $\begin{array}{l}\text { Groupe I } \\
\text { n (\%) }\end{array}$ & $\begin{array}{l}\text { Groupe II } \\
\text { n (\%) }\end{array}$ & Valeur p \\
\hline Score de Glasgow & & $0(0)$ & \\
$3-4$ & $0(0)$ & $0(0)$ & \\
$5-12$ & $0(0)$ & $15(100)$ & \\
$13-15$ & $17(100)$ & $04(26.67)$ & 0.0131 \\
Score NIHSS & $12(70.58)$ & $08(53.33)$ & \\
\hline$<10$ & $05(23.52)$ & $03(20)$ & \\
$10-15$ & $0(0)$ & $0(0)$ & \\
$16-20$ & $0(0)$ & $04(26.67)$ & \\
$>20$ & & $07(46.67)$ & \\
Localisation & $07(41.18)$ & $01(6.66)$ & \\
saignement & $07(41.18)$ & $03(20)$ & \\
Lobaire & $01(5.88)$ & & \\
Noyaux gris centraux & $02(11.76)$ & $7(46.66)$ & \\
Cervelet & & $8(53.33)$ & \\
Tronc cérébral & $7(41.17)$ & & \\
Signe d'engagement & $10(58.82)$ & & \\
Oui & & & \\
Non & & & \\
& & & \\
\hline
\end{tabular}

Une association a été décrite entre l'HTA à la phase aiguë de l'AVC et un score NIHSS > 10 (Tableau III). 
Tableau III. Comparaison des 02 groupes selon les données cliniques

\begin{tabular}{|l|l|l|l|}
\hline & $\begin{array}{l}\text { Groupe I } \\
\text { n (\%) }\end{array}$ & $\begin{array}{l}\text { Groupe I } \\
\text { n (\%) }\end{array}$ & Valeur p \\
\hline Valeur TA systolique J3 & $0(0)$ & $4(26.67)$ & \\
$\geq 180$ & $11(64.70)$ & $10(66.67)$ & 0.0506 \\
$140-179$ & $06(35.30)$ & $01(6.66)$ & \\
$110-139$ & & & \\
Valeur TA systolique J7 & $0(0)$ & $01(6.66)$ & \\
\hline$\geq 180$ & $06(35.30)$ & $07(46.67)$ & 3.137 \\
$140-179$ & $11(64.70)$ & $05(33.33)$ & \\
$110-139$ & & & \\
Survenue de décès & $0(0)$ & $0(0)$ & \\
\hline J1-J3 & $0(0)$ & $03(20)$ & \\
J4-J7 & & $0(0)$ & \\
Score ICH* & $0(0)$ & $9(60)$ & \\
\hline $\mathbf{0}$ & $12(70.58)$ & $3(20)$ & \\
$\mathbf{1}$ & $4(23.52)$ & $3(20)$ & \\
$\mathbf{2}$ & $1(5.88)$ & $0(0)$ & \\
$\mathbf{3}$ & $0(0)$ & $0(0)$ & \\
$\mathbf{4}$ & $0(0)$ & & \\
$\mathbf{5}$ et 6 & $0(1)$ & & \\
\hline
\end{tabular}

*Score ICH : Intra-Cerebral Hemorrhage Score

Trois patients appartenant au groupe II (20\%) ont succombé pendant la phase aiguë de leur AVC. Un patient a eu une TAS à l'admission entre $180-220 \mathrm{mmHg}$ et deux patients avec une TAS supérieure ou égale à $220 \mathrm{mmHg}$.

\section{Discussion}

Nos résultats ont rapporté $20 \%$ (3/15) de mortalité à la phase aiguë dans le groupe II (patients avec TAS à l'admission supérieur ou égale à 180 $\mathrm{mmHg}$ ) sous notre protocole de prise en charge de l'HTA à la phase aiguë de l' AVC hémorragique et pas de décès rapporté $(0 / 17)$ dans le groupe I (patients avec TAS à l'admission entre $140-179 \mathrm{mmHg}$ ). L'objectif TAS à J7 de moins de $140 \mathrm{mmHg}$ n'a pas été atteint dans les deux groupes de populations soit dans $35.29 \%$ des cas du groupe I $(n=6)$ et dans $46.66 \%$ des cas du groupe II $(n=7)$.

L'hypertension artérielle (TA $>140 / 90 \mathrm{mmHg}$ ) à la phase aiguë d'un AVC hémorragique touche approximativement $75 \%$ des patients (Qureshi, 2007). Elle résulte de plusieurs mécanismes et représente à la fois la cause et la conséquence de la lésion. Cette élévation de la TA à la phase aiguë est d'une part associée à un risque accrue de resaignement, d'expansion de l'hématome et de l'œdème péri lésionnel et peut être aussi associée à une altération précoce de l'état neurologique (33\% des cas dans les 48h) (Balami, 2012). D'autre part, elle constitue un des facteurs de mortalité intra hospitalière (Stapf, 2015). Cette mortalité précoce est de l'ordre de 30 à $50 \%$, dont la moitié survient dans les premières $48 \mathrm{~h}$ de l'AVC (Broderick, 2001). Cette donnée de la littérature est concordante avec notre résultat. 
Peu sont les stratégies thérapeutiques mises en œuvre devant une hémorragie intra parenchymateuse face aux échecs successifs des thérapeutiques hémostatiques, neuroprotectrices. Et comme rapporte le papier de Rosso C (Rosso, 2016) sur l'HTA à la phase aiguë, contrôler la tension artérielle est une façon de se dire que les équipes font «quelque chose ». La Société Française Neuro-Vasculaire (SFNV) (Stapf, 2015), devant des patients avec une hémorragie intra parenchymateuse sépare leur recommandation selon deux groupes de patients : D'un côté ceux qui ont une TAS initiale entre $150-220 \mathrm{mmHg}$ et de l'autre côté ceux avec une TAS initiale $>220 \mathrm{mmHg}$. Elle recommande que les patients ayant une hémorragie cérébrale de moins de 6 heures, non traumatique, non malformative (spontanée), et une TAS entre 150-220 mmHg, peuvent bénéficier d'une baisse rapide de la TAS en moins de 60 minutes, avec une TA cible inférieure à $140 \mathrm{mmHg}$. Cette baisse est réalisable, bien tolérée et probablement efficace sur le pronostic fonctionnel. Cette baisse doit être précoce, et la réduction du niveau de la TA $(<140$ $\mathrm{mmHg}$ ) obtenue dans l'heure suivant la mise en place du traitement est maintenue durant au moins une semaine. Aucune donnée n'est disponible pour les patients ayant une TAS initiale supérieure à $220 \mathrm{mmHg}$. Et elle ajoute aussi concernant l'agent hypotenseur utilisé « aucun agent n'a été spécifiquement étudié ». Néanmoins, l'Urapidil et la Nicardipine par voies IV semblent facilement utilisables dans le cadre d'une Unité de Soins Intensifs NeuroVasculaire (USINV) (Stapf, 2015).

L'application de cette recommandation se base sur trois paramètres très importants : le patient devrait avoir une preuve radiologique de la nature hémorragique de son $\mathrm{AVC}$, ceci à moins de $6 \mathrm{~h}$ du début de ses symptomatologies et se faire hospitaliser dans une Unité de Soins de Neurologie Vasculaire. Dans notre Hôpital Universitaire Befelatanana Antananarivo, on se heurte à trois grands problèmes. Premièrement, d'après l'étude de Raveloson NE et al en 2011 sur 34 cas de patients vus auprès du service ATU HU Befelatanana, $45 \%$ des patients arrivent à plus de six heures du début de ses symptômes et 12 patients $(35,29 \%)$ seulement des centres de santé publique ou des cabinets médicaux privés sont référés. Deuxièmement, pour que le patient ait son scanner cérébral (qui est à sa charge et coûte- en moyenne 40 euros -représentant le salaire moyen d'un malgache) dans les meilleures conditions, il faudra compter une heure de temps même s'il arrive à moins de $6 \mathrm{~h}$, car l'hôpital ne dispose pas de scanner cérébral au sein de sa structure. Ceci démontre du manque d'Information, d'Education et de Communication (IEC) sur l'AVC autant chez la population générale que chez les acteurs de la Santé. De ce fait qu'il soit nécessaire la mise en place la filière AVC à Madagascar, tout au moins la vulgarisation de la nécessité de se faire hospitaliser en cas de signes en faveur de l'AVC. Troisièmement, il n'existe pas encore d'USINV à Madagascar qui soit habilitée à prendre en 
charge les AVC à la phase aiguë. Dans notre hôpital, les patients sont orientés selon leur état de vigilance (basé sur le score de Glasgow) et aussi du résultat du scanner cérébral si on en dispose. Les patients avec un score de Glasgow < 13 restent hospitaliser auprès du Service de Réanimation Urgence, ceux dont le score de Glasgow $\geq 13$ avec des bons paramètres cliniques sont orientés directement auprès du Service de Neurologie (patients sélectionnés dans notre étude).

Notre protocole de prise en charge de l'HTA à la phase aiguë de l'AVC hémorragique se base sur les données des études multicentriques randomisées. La moitié de nos patients n'ont pu faire le scanner cérébral qu'entre la 48è et 72 è heures de leur hospitalisation $(n=16)$. Selon les données de l'étude ATACH-2 en 2016 (Qureshi, 2016), sur 1000 patients ayant un AVC hémorragique en moins de 4,5heures et une TAS comprise entre 180-240 $\mathrm{mmHg}$, deux groupes de population ont été comparés : ceux dont la TAS a été maintenue entre $140-179 \mathrm{mmHg}(\mathrm{n}=500)$ et ceux avec une stratégie intensive 110-139 $\mathrm{mmHg}$, ceci en utilisant du Nicardipine IV maintenue pendant $24 \mathrm{~h}$ à $5 \mathrm{mg} / \mathrm{h}$. Si la TA demeure en-dessus des cibles après 30 minutes, le Labetalol IV était utilisé, sinon le Diltiazem ou l'Urapidil en IV. Elle ne rapporte pas de différence significative sur le taux de mortalité / handicap sévère $(\mathrm{p}=0.72)$. Une méta analyse, faite par l'équipe de Tsivgoulis et al en 2014 sur quatre études (ICH ADAPT, INTERACT I, INTERACT II, Kock et al) incluant 3315 patients, a rapporté un taux de mortalité à 3 mois similaire après randomisation des patients mise sous une stratégie intensive contre ceux suivant la recommandation standard de la baisse de la tension artérielle à la phase aiguë de l'AVC (OR 1.01, 95\% IC 0.83 à $1.23 ; \mathrm{p}=0.914)$. Ainsi, tant que nos patients n'ont pas réalisé le scanner cérébral, nos objectifs tensionnels systoliques étaient de moins de $180 \mathrm{mmHg}$. La Nicardipine par voie IV était notre anti hypertenseur pendant les quatre premiers jours de l'AVC hémorragique (période pendant laquelle il y a une perte de l'autorégulation de la perfusion cérébrale) et la perfusion est maintenue pendant la première semaine. S'il y a fluctuation de la tension artérielle, l'adjonction d'anti hypertenseur oral sera laissée au choix du neurologue responsable. L'objectif de la TAS à $\mathrm{J} 7$ était inférieur à $140 \mathrm{mmHg}$. Le choix de la durée de sept jours nous vient des données de l'étude INTERACT II de Anderson et al en 2013, sur 2839 patients ayant un AVC hémorragique de moins de 6 heures et une TAS systolique à l'admission entre $150-220 \mathrm{mmHg}$. Cette dernière étude a comparé deux groupes de population : ceux dont la TAS a été maintenue $<180 \mathrm{mmHg}(\mathrm{n}=1430)$ et ceux avec une stratégie intensive $<140 \mathrm{mmHg}$ (qui devrait être atteinte en moins d'une heure et maintenue pendant $7 \mathrm{j})(\mathrm{n}=1399)$. L'étude a aussi rapporté une réduction significative du risque de décès / handicap de $13 \%(\mathrm{p}=0.044)$. L'Urapidil a été l'anti HTA utilisé dans plus de $30 \%$ des cas pour le groupe avec la stratégie intensive, 
suivi par Nicardipine ou Nimodipine 16.2\%, Labetalol 14.4\%, Nitroglycérine 14.9\%, Furosémide 12.4, Nitroprusside 12.1\%, Hydralazine 5.9\% (Anderson, 2013).

Comme le Service de Neurologie ne dispose pas encore d'Unité de Soins Intensifs en Neurologie Vasculaire (USINV), quatre lits sont réservés auprès du service pour les AVC transférés à J1 par l'ATU. Les patients y sont surveillés toutes les heures et, si nécessaire pendant la mise sous scope, et ils sont sous surveillance tensionnelle étroite s'il y a une mise sous protocole Nicardipine IV.

Nous avons choisi de décrire le taux de mortalité dans les deux groupes de notre population d'étude à $\mathrm{J} 3$ et $\mathrm{J} 7$ par rapport à notre protocole pour baisser la TA à la phase aiguë, ceci car nous avons été limités par le temps. Cependant, ces différentes études randomisées évaluent surtout la mortalité et le handicap des patients à 90 jours.

Une autre étude analytique ultérieure type cohorte devrait être réalisée pour évaluer l'efficacité de ce protocole de baisse de la tension artérielle à la phase aiguë, la survie et le handicap à 90j de ces patients.

La création d'une filière AVC devrait être faite à Madagascar avec la formation de tous les acteurs de la santé y afférent. Il faudrait aussi munir l'HU Befelatanana d'une USINV capable de prendre en charge correctement nos patients atteints d'AVC.

Néanmoins, d'autres paramètres peuvent aggraver aussi le pronostic vital et fonctionnel des patients neurolésés que nous n'avons pas établir dans notre étude, mise à part 1'HTA à la phase aiguë (Grossac, 2010). Ces paramètres pourraient être d'origine intra crânienne (hématome, hypertension intra crânienne, ...) ou extra crânienne (hypoxémie, valeur de la capnie, fièvre) regroupés sous le nom d'agressions cérébrales d'origine systémique (ACSOS). De la même manière, une échelle de gravité permettant d'établir des valeurs seuils a été développée par l'Université d'Edimbourg (Jones, 1994), ce qui pourrait créer un biais de confusion lors des interprétations de nos résultats.

Dans l'étude de Raveloson et al en 2011 à Madagascar, l'âge moyen de la population était de 57 ans (38-78) avec une prédominance féminine et un sex ratio de 0,78. Dans celle de Qureshi et al en 2016 dans 110 sites internationaux (ATACH II) qui a recruté 1000 (4 ans et 4mois) avec (500 / 500) ceux avec un traitement agressif avaient un âge moyen de 62+/- 13.1 ans et ceux avec traitement standard avaient $61.9+/-13.1$ ans avec une prédominance masculine à $304(60.8 \%)$ et $316(63.2 \%)$ respectivement. Anderson CS et al en 2013 dans 144 sites en 21 pays avec 2829 (1399/ 1430) retrouvait que ceux avec un traitement agressif avait un âge moyen de $63+/$ 13.1 ans et ceux avec traitement standard avaient $64.1+/-14.6$ ans avec une prédominance masculine avec $898(64.2 \%)$ et $882(61.7 \%)$ respectivement. 
L'étude de Yamada T et al 2017 au Japon retrouvait que ceux avec un traitement agressif avait un âge moyen de 63+/- 13.1ans et ceux avec traitement standard à bon pronostic avaient $66.9+/-11.1$ ans et ceux de mauvais pronostic avaient 72.2+/- 13.3 ans, à prédominance masculine aussi avec 199 / 126 et 198 / 181 dans les deux groupes et finalement notre étude en 2017, à Madagascar qui montre 32 / 729 (12 mois) avec 59.1ans (24 à 72) et un sex ratio 1 . Notre population d'étude est de taille très réduite comparée à la littérature à propos de ce sujet. Cela s'explique par la courte durée de notre recrutement ainsi que par notre critère de sélection des patients. L'âge moyen de notre population d'étude est plus jeune comparé aux données de la littérature, conforme à une étude Malagasy sur 1'AVC des artères perforantes de Rasaholiarison NF et al. (2017), laquelle a rapporté un âge médian de 59.09 ans et dont $59.03 \%$ avaient moins de 60 ans.

L'HTA est un grand facteur de risque de survenue d'un AVC hémorragique. Plus elle est élevée, plus le risque de développer un AVC hémorragique est élevée, comme ce qui a été démontré par deux études de cohorte fait par Leppälä et al (RR ajusté 2.20 pour TA 140-159 mmHg et 3.78 pour TA $\geq 160 \mathrm{mmHg}$ comparé à $\mathrm{TA} \leq 139 \mathrm{mmHg}$ ) et celle de Suh et al (RR à 2.2 si TA normale haute, 5.3 HTA stade I, 10.4 stade II et 33 stade III).

Une étude dans le cadre de «the Honolulu Heart Program » a suivi pendant 12 ans, 8006 hommes (2916 non alcooliques et 4962 alcooliques). Ils ont rapporté que l'alcoolisme est un facteur de risque de survenue indépendant de l'HTA dans la survenue de l'AVC hémorragique. Il double la survenue de l'AVC hémorragique chez les alcooliques modérés et le triple chez les grands buveurs (Donahue, 1986), ce qui est le cas dans notre étude.

Dans l'étude de Raveloson et al en 2011 à Madagascar avec un nombre de cas de 34 patients, sur 12 mois, les scores de Glasgow étaient répartis en ]13-15] : 0 (0\%), entre ]08-13] 16 (47.05\%) et entre [03-08] 18 (52.94\%). Qureshi et al en 2016 sur 110 sites internationaux (ATACH II) avec ( $\mathrm{N}=$ 1000), sur 4ans et 4 mois. Dans le groupe avec TA $110-139 \mathrm{mmHg}$, un score à 15 avec 275 cas $(55.0 \%)$, un score entre $12-14$ avec 152 cas $(30.4 \%)$, entre $3-11$ avec 73 cas $(14.6 \%)$ et dans le groupe $149-179 \mathrm{mmHg}$ pour chaque intervalle de score avec 284 cas (56.8\%), 142 cas (28.4\%) et 74 cas(14.8), avec un score NIHSS moyen à $11(0-40)$. Dans l'étude de Yamada T et al en 2017 avec 704 patients, sur 4 ans, dans 2 groupes de bon pronostic avec un score de Glasgow à 14-15 composé de 265 patients, entre $9-13$ avec 51 patients, entre 3-8 avec 9 patients et dans le groupe de mauvais pronostic avec $158 \mathrm{cas}$, avec 138 cas, avec 83 cas. Dans l'étude de Anderson CS et al en 2013, sur 144 sites, dans 21 pays (INTERACT II) avec 2829 patients dans 4 groupes, dans le groupe <140 mmHg avec un score de Glasgow à 14 (12 -15), puis dans le groupe <180 mmHg à 14 (12 -15), puis <140 mmHg à 10 (6-15), enfin dans $<180$ mmHg 11 (6-16). Dans notre étude en 2017 à Madagascar avec 32 
patients, sur 12 mois, le score de Glasgow était à 13-15 dans les 2 groupes, le score de NIHSS dans le groupe I était <10 (12\%) et entre 10-15 (4\%), et dans le groupe II un score $<10$ (4\%), entre 10-15 (8\%), entre 16-20 (3\%). Une signification a été décrite entre l'HTA à la phase aiguë de l'AVC et un score NIHSS > 10. Dans l'étude de Anderson CS et al avec 2794 patients, le TDM cérébral était à $<6$ heures dans ces 2 groupes avec localisation profonde 1084 $(83.8 \%)$ dans le premier groupe et 1098 (83.2\%) dans le deuxième. Pour Qureshi et al ( $\mathrm{N}=1000)$, le scanner est à $<4.5$ heures, avec localisation lobaire avec 104 patients et au niveau des noyaux gris centraux (NGC) avec 845 patients. Dans notre étude avec 32 patients, dans le groupe I, les temps du scanner cérébral étaient 5 (29.41\%) à J1, 8 (47.05\%) à J2-J3, 4 (23.52\%) à J4$\mathrm{J} 7$ et dans le groupe II 5 (33.33\%), 8 (53.33\%), 2 (13.33\%). Les localisations lobaires, NGC, cervelet, tronc cérébral étaient respectivement à $7(41.17 \%), 7(41.17 \%), 1(5.88 \%)$ et $2(11.76 \%)$ dans le groupe I et dans le groupe II à $4(26.66 \%), 7(46.66 \%), 1(6.66 \%)$ et $3(20 \%)$. Le score ICH à 1 , 2,3 avec $12(70.58 \%), 4(23.52 \%)$ et 1 (5.88) dans le groupe I et $9(60 \%), 3$ $(20 \%)$ et $3(20 \%)$ dans le groupe II. L'ICH score est un score facile à calculer, permettant d'évaluer la mortalité à 30 jours et le pronostic fonctionnel à 12 mois (Kim, 2017). Nous n'avons pas trouvé d'association avec l'élévation de la TAS à la phase aiguë, cependant, l' ICH score seul ne permet pas de juger de la mise ou non sous traitement à la phase aiguë de l'AVC. Dans les études INTERACT II (Anderson, 2017) et ATACH II (Qureshi, 2016), les patients ont pu bénéficier d'un scanner cérébral pour la confirmation de la nature anatomique de leur AVC respectivement en moins de $6 \mathrm{~h}$ et de $4.5 \mathrm{~h}$. Le scanner cérébral reste le «gold standard » pour le diagnostic de l'AVC hémorragique à la phase aiguë. Il permet de caractériser l'hématome (localisation, volume), ses complications (effets de masse, inondation intra ventriculaire) et, après injection de produit de contraste, son étiologie (MAV, anévrysme, cavernome ...) et le risque d'expansion hémorragique par la présence d'extravasation du produit de contraste (spot sign). Vu les circonstances, il serait mieux de doter l'HU Befelatanana d'un scanner cérébral pour que les patients qui arrivent en début de leur AVC puissent bénéficier de cet examen.

\section{Conclusion}

Notre travail a mis en évidence une mortalité intra hospitalière à la phase aiguë de l'AVC hémorragique qui reste élevée, et la négligence de la prise en charge de l'HTA par nos patients. D'ici la nécessité de mettre en place la filière AVC à Madagascar et d'en créer les structures sanitaires y afférent, notamment une Unité de Soins Intensifs de Neurologie Vasculaire et un scanner cérébral à faible coût disponible 24 heures/24 pour les patients avec un AVC. Il est recommandable aussi de former les personnels médicaux qualifiés dans ses tâches. 


\section{References:}

1. Anderson, CS., Heeley, E., Huang, Y., Wang, J., Stapf, C., Delcourt, $\mathrm{C}$ et al. INTERACT2 Investigators. (2013). Rapid blood-pressure lowering in patients with acute intracerebral hemorrhage. $\mathrm{N}$ Engl J Med $20 ; 368(25): 2355-65$.

2. Balami, JS., \& Buchan, AM. (2012). Complications of intracerebral haemorrhage. Lancet Neurol ; 11: 101-18.

3. Broderick, J., Connolly, S., Feldmann, E., Hanley, D., Kase, C., Krieger, D., et al. (2007). Guidelines for the management of spontaneous intracerebral hemorrhage in adults: 2007 update: a guideline from the American Heart Association/American Stroke Association Stroke Council, High Blood Pressure Research Council, and the Quality of Care and Outcomes in Research Interdisciplinary Working Group. Stroke ; 38 : 2001-23.

4. Chu, S., \& Sansing, L. (2017). Evolution of blood pressure management in acute intracerebral hemorrhage [version 1; referees: 4 approved]. F1000 research, 6 (F 1000 Faculty Rev) : 2035 (doi: 10.12688/f1000research.11687.1).

5. Davis, SM., Broderick, J., Hennerici, M., et al. (2006). Hematoma growth is a determinant of mortality and poor outcome after intracerebral hemorrhage. Neurology ; 66 : 1175- 81.

6. Donahue, Rp., Abbot, RD., Reed, DM. et al. (1986). Alcohol and hemorrhagic stroke : The Honolulu Heart Program. JAMA ; 255 (17) :2311-4.

7. Feigin, VL., Lawes, CM., Bennett, DA., \& Anderson, CS. (2003). Stroke epidemiology : a review of population-based studies of incidence, prevalence, and case-fatality in the late 20th century. Lancet Neurol ; 2 : 43-53.

8. Grossac, J., Fourcade, O., \& Geeraerts, T. (2010). AVC hémorragique aux urgences (hors hémorragie méningée). 52e congrès national d'anesthésie et de réanimation Médecins. Urgences vitales. Sfar. Pages 1-12.

9. Jones, PA., Andrews, PJ., Midgley, S., Anderson, SI., Piper, IR., Tocher, JL., et al. (1994). Measuring the burden of secondary insults in head-injured patients during intensive care. J Neurosurg Anesthesiol ; $6: 4-14$.

10. Kim, JY., \& Bae, HJ. (2017). Spontaneous Intracerebral Hemorrhage: Management. Journal of Stroke ;19 (1) : 28-39.

11. Kumar, S. (2017). Hypertension and hemorrhagic stroke. Hypertension Journal ; 3(2) : 89-93. 
12. Organisation Mondiale de la Santé. Politique Nationale de Prévention et Lutte Intégrées Contre les Maladies Chroniques Non Transmissibles. Page

13. https://extranet.who.int/nutrition/gina/sites/default/files/MDG-2013PNLMCNT.pdf

14. Ohwaki, K., Yano, E., Nagashima, H., Hirata, M., Nakagomi, T., \& Tamura, A. (2004). Blood pressure management in acute intracerebral hemorrhage: relationship between elevated blood pressure and hematoma enlargement. Stroke ; 35(6) :1364-7.

15. Qureshi, AI., Tuhrim, S., Broderick, JP., et al. (2001). Spontaneous intracerebral hemorrhage. N Engl J Med ; 344 : 1450-60.

16. Qureshi, A.I. (2013). The importance of acute hypertensive response in ICH. Stroke 44 : S67-69.

17. Qureshi, AI., Ezzeddine, MA., Nasar, A., et al. (2007). Prevalence of elevated blood pressure in 563,704 adult patients with stroke presenting to the ED in the United States. Am J Emerg Med ; 25 : 32 8.

18. Qureshi, AI., Palesch, YY., Barsan, WG. et al. (2016). Intensive Blood-pressure lowering in patients with acute cerebral hemorrhage. N Engl J Med ; 375 (11) : 1033-43.

19. Rasaholiarison, NF., Rahamefy, RO., Rajaonarison, LA. et al. (2017). Fréquence et caractéristiques des AVC impliquant les artères perforantes dans le Service de Neurologie de l'Hôpital Befelatanana, Antananarivo. Pan Af Med J ; 28:76-81.

20. Ravelojaona, FH. (2012). Etude épidémio-économique des Accidents Vasculaires Cérébraux. Thèse de Doctorat. Page 23.

21. Raveloson, NE., Zodaly, N., Rakotoarivony, ST., Mbolamena, RL., \& Randriamiarana, JM. (2011). Aspects épidémiocliniques, évolutifs et tomodensitométriques des accidents vasculaires cérébraux hémorragiques (34 cas). Revue d'Anesthésie-Réanimation et de Médecine d'Urgence ; 3(1): 15-9.

22. Rosso, C. (2016). Le point sur l'hypertension artérielle à la phase aiguë de l'AVC. Etre strict sur l'objectif est-il bénéfique pour le patient. CORDIAM ; page : 26-28.

23. Stapf, C., Béjôt, Y., Tardy, J., \& Cordonnier, C. (2015). Pression artérielle à la phase aiguë des hemorragies cérébrales. Préconisation de la SFNV ; pages1-5.

24. Steiner, T., Diringer, MN., Schneider, D., et al. (2006). Dynamics of intraventricular hemorrhage in patients with spontaneous intracerebral hemorrhage: risk factors, clinical impact, and effect of hemostatic therapy with recombinant activated factor VII. Neurosurgery ; 59 : 767-73. 
25. Tsivgoulis, G., Katsanos, AH., Butcher, KS. et al. (2014). Intensive blood pressure reduction in acute intracerebal hemorrhage : a metaanalysis. Neurology ; $83: 1532-9$.

26. Vuillier, F., Medeiros-De-Bustos, E., \& Moulin, T. (2004). Exploration d'un déficit neurologique. J.Neuroradiol ; $31: 252-61$.

27. Yamada, T., \& Natori, Y. (2017). Acute blood-pressure management and prognostic factors in patients with intracerebral hemorrhage. Interdisciplinary Neurosurgery : Advanced Techniques and case Management ; $10: 91-5$. 